\title{
ON SOME APPROXIMATION PROBLEMS IN METRIC SPACES
}

\author{
T.D. NARANG
}

In this paper we consider the problem of simultaneous characterization of a set of elements of best approximation and characterization of elements of $\varepsilon$-approximation in metric spaces.

\section{Introduction}

The theory of best approximation in metric spaces is comparatively less developed than that in normed linear spaces or linear metric spaces. Only a few mathematicians have pursued this study in sporadic attempts. The author in a series of papers has also made an attempt in this direction and the present paper is also a step in the same direction.

In this paper we discuss the problem of simultaneous characterization of a set of elements of best approximation from which follows a characterization of semi-Chebyshev subspaces and also give a characterization of elements of $\varepsilon$-approximation in metric spaces.

\section{Simultaneous characterization of set of elements of best approximation}

The problem of characterization of elements of best approximation in metric spaces was considered by Mustăta [3]. A natural generalization of this is the following problem of simultaneous characterization of a set of elements of best approximation:

Given a non-empty subset $Y$ of a metric space $(X, d), x \in X \backslash Y$ and a subset $M$ of $Y$, what are the necessary and sufficient conditions in order that every element $y_{0} \in M$ be an element of best approximation to $x$ by the elements of $Y$ ?

We shall answer this in Theorems 2.2 and 2.3. As a consequence of Theorem 2.2, we get a characterization of semi-Chebyshev subspaces (Corollary 2.1).

Let $(X, d)$ be a metric space and $x_{0}$ a fixed point of $X$. The set

$$
X_{o}^{\#}=\left\{f: X \longrightarrow \mathbb{R}, \sup _{x, y \in X x \neq y} \frac{|f(x)-f(y)|}{d(x, y)}<\infty, f\left(x_{0}\right)=0\right\},
$$

of Lipschitz functions on $X$ vanishing at $x_{o}$, is a Banach space (even a conjugate Banach space-see Johnson [2]) with the usual operations of addition, multiplication by real scalars

Received February 24, 1987. 
and normed by

$$
\|f\|_{X}=\sup _{x, y \in X x \neq y} \frac{|f(x)-f(y)|}{d(x, y)}, f \in X_{0}^{\#} .
$$

If $Y$ is a subset of a metric space $(X, d)$ and $x \in X$ then an element $y_{0} \in Y$ is said to be an element of best approximation of $x$ by elements of $Y$ if $d\left(x, y_{0}\right)=d(x, Y)$. We shall denote by $L_{Y}(x)$ the set of all best approximations to $x$ in $Y$. The set $Y$ is said to be semi-Chebyshev if $L_{Y}(x)=\phi$ or singleton for each $x \in X$.

Mustăta [3] gave the following characterization of elements of best approximation in metric spaces. A similar characterization in normed linear spaces was obtained by Singer (see [7]) and in linear metric spaces by Pantelidis [6].

Theorem 2.1. Let $Y$ be a subset of a metric space $(X, d)$ such that $x_{0} \in Y$ and let $x \in X \backslash Y$. Then $y_{0} \in Y$ is an element of best approximation for $x$ by elements of $Y$ if and only if there is an $f \in X_{0}^{\#}$ such that

(i) $\|f\|_{X}=1$

(ii) $\left.f\right|_{Y}=0$

(iii) $\left|f(x)-f\left(y_{o}\right)\right|=d\left(x, y_{0}\right)$.

The following theorem gives simultaneous characterization of a set of elements of best approximation in metric spaces. In normed linear spaces a similar characterization was given by Singer (see [7]) and in linear metric spaces by Narang and Khanna [5].

Theorem 2.2. Let $Y$ be a subset of a metric space $(X, d)$ such that $x_{0} \in Y$ and let $x \in X \backslash Y$ and $M \subset Y$. Then $M \subset L_{Y}(x)$ if and only if there exists an $f \in X_{o}^{\#}$ such that (a) $\|f\|_{X}=1$

(b) $\left.f\right|_{Y}=0$

(c) $|f(x)-f(y)|=d(x, y)$ for all $y \in M$.

Proof. Suppose that $M \subset L_{Y}(x)$ and $y_{0} \in M$. Then $y_{0} \in L_{Y}(x)$ and so by Theorem 2.1, there exists an $f \in X_{0}^{\#}$ satisfying (a),(b) and $\left|f(x)-f\left(y_{0}\right)\right|=d\left(x, y_{0}\right)$.

Now, let $y \in M$. Then $y \in L_{Y}(x)$ i.e. $d(x, y)=d(x, Y)=d\left(x, y_{0}\right)$. Consider

$$
\begin{aligned}
|f(x)-f(y)| & =\left|f(x)-f\left(y_{o}\right)\right| \text { as }\left.f\right|_{Y}=0 \\
& =d\left(x, y_{0}\right) \\
& =d(x, y) .
\end{aligned}
$$

Thus (c) is also satisfied.

Conversely, suppose that there exists an $f \in X_{o}^{\#}$ satisfying (a),(b) and (c) and let $y_{0} \in M$. Then by Theorem 2.1, $y_{0} \in L_{Y}(x)$. Hence $M \subset L_{Y}(x)$.

As a consequence of Theorem 2.2, we get the following characterization of semiChebyshev subspaces of metric spaces.

Corollary 2.1. Let $Y$ be a subset of a metric space $(X, d)$ such that $x_{0} \in Y$. Then the following statements are equivalent: 
(i) $Y$ is semi-Chebyshev

(ii) There exist no $f \in X_{0}^{\#}, x_{1} \in X$ and $y_{1}, y_{2} \in Y, y_{1} \neq y_{2}$ such that

(a) $\|f\|_{X}=1$

(b) $\left.f\right|_{Y}=0$

(c) $f\left(x_{1}\right)=d\left(x_{1}, y_{1}\right)=d\left(x_{1}, y_{2}\right)$.

An independent proof of this result was given by Mustăta [4].

Let $Y$ is a subset of a metric space $(X, d)$ and $x_{o} \in Y$. If

$$
Y^{\perp}=\left\{f \in X_{0}^{\#}:\left.f\right|_{Y}=0\right\},
$$

and

$$
d_{Y^{\perp}}(x, y)=\sup _{f \in Y^{\perp}\{0\}} \frac{|f(x)-f(y)|}{\|f\|_{X}}, x, y \in X
$$

we have the following inequality (see [3]):

$$
d_{Y \perp}(x, y) \leq d(x, y) \text { for all } x, y \in X .
$$

The following characterization of elements of best approximation was given by Mustăta [3]:

Theorem 2.3. Let $Y$ be a subset of a metric space $(X, d)$ such that $x_{o} \in Y$ and let $x \in X \backslash Y$. Then $y_{o} \in Y$ is an element of best approximation for $x$ by elements of $Y$ if and only if

$$
d_{Y \perp}\left(x, y_{0}\right)=d\left(x, y_{o}\right) .
$$

Using Theorem 2.2, we get another result on the simultaneous characterization of a set of elements of best approximation in metric spaces from which Theorem 2.3 follows as a corollary.

Theorem 2.4. Let $Y$ be a subset of a metric space $(X, d)$ such that $x_{o} \in Y$ and let $x \in X \backslash Y$ and $M \subset Y$. Then $M \subset L_{Y}(x)$ if and only if

$$
d_{Y^{\perp}}(x, y)=d(x, y)
$$

for all $y \in M$.

Proof. Let $M \subset L_{Y}(x)$. Then by Theorem 2.2 there exists an element $f \in Y^{\perp}$ such that $\|f\|_{X}=1$ and $|f(x)-f(y)|=d(x, y)$ for all $y \in M$. We have

$$
d_{Y \perp}(x, y)=\sup _{g \in Y^{\perp}\{\{\}} \frac{|g(x)-g(y)|}{\|g\|_{X}} \geq \frac{|f(x)-f(y)|}{\|f\|_{X}}=d(x, y)
$$

and as $d_{Y^{\perp}}(x, y) \leq d(x, y)$ for all $x, y \in X$, we have $d_{Y^{\perp}}(x, y)=d(x, y)$ for all $y \in M$. 
Conversely, suppose $d_{Y \perp}(x, y)=d(x, y)$ for all $y \in M$. Then for any $y_{0} \in M$ and $z \in Y$ we have

$$
\begin{aligned}
d\left(x, y_{0}\right) & =d_{Y \perp}\left(x, y_{0}\right) \\
& =\sup _{f \in Y^{\perp} \backslash\{0\}} \frac{\left|f(x)-f\left(y_{0}\right)\right|}{\|f\|_{X}} \\
& =\sup _{f \in Y^{\perp} \backslash\{0\}} \frac{|f(x)-f(z)|}{\|f\|_{X}} \\
& =d_{Y \perp}(x, z) \\
& =d(x, z)
\end{aligned}
$$

Hence $y_{0} \in L_{Y}(x)$ i.e. $M \subset L_{Y}(x)$.

\section{A characterization of elements of $\varepsilon$-approximation}

Let $Y$ be a subset of a metric space $(X, d), x \in X$ and $\varepsilon>0$. An element $y_{0} \in Y$ is said to be an element of $\varepsilon$-approximation of $x$ by elements of $Y$ if $d\left(x, y_{0}\right) \leq d(x, Y)+\varepsilon$. We shall denote by $L_{Y}(x, \varepsilon)$, the set of all elements of $\varepsilon$-approximation to $x$. In particular, for $\varepsilon=0$ we find again the elements of best approximation of $x$ and respectively the set $L_{Y}(x)$. The concept of $\varepsilon$-approximation was introduced by R.C. Buck [1] who used the term 'elements of good approximation'. Buck [1] and Singer (see [7]) characterized elements of $\varepsilon$-approximation in normed linear spaces. In linear metric spaces these elements were characterized by Narang and Khanna [5]. Here we give a characterization of such elements in metric spaces (Theorem 3.1). In the particular case when $\varepsilon=0$, Theorem 3.1 reduces to Theorem 2.1 on the characterization of elements of best approximation.

Theorem 3.1. Let $Y$ be a subset of a metric space $(X, d)$ such that $x_{0} \in Y$ and let $x \in X \backslash Y, y_{0} \in Y$ and $\varepsilon>0$. Then $y_{0} \in L_{Y}(x, \varepsilon)$ if and only if there exists an $f \in X_{0}^{\#}$ such that

(i) $\|f\|_{x}=1$

(ii) $\left.f\right|_{Y}=0$

iii) $\left|f(x)-f\left(y_{0}\right)\right| \geq d\left(x, y_{0}\right)-\varepsilon$.

Proof. Suppose $y_{0} \in L_{Y}(x, \varepsilon)$. Define $f(z)=d(z, Y), z \in X$. Then from the proof of Proposition 1 [3] it follows that this function satisfies (i), (ii) and

$$
\begin{aligned}
\left|f(x)-f\left(y_{0}\right)\right| & =d(x, Y) \\
& \geq d\left(x, y_{0}\right)-\varepsilon .
\end{aligned}
$$

Conversely, let us suppose that there exists $f \in X_{0}^{\#}$ satisfying (i), (ii) and (iii). 
Then

$$
\begin{aligned}
d\left(x, y_{0}\right) & \leq\left|f(x)-f\left(y_{0}\right)\right|+\varepsilon \\
& =|f(x)-f(y)|+\varepsilon \text { for all } y \in Y \\
& \leq\|f\|_{X} d(x, y)+\varepsilon \text { for all } y \in Y \\
& =d(x, y)+\varepsilon \text { for all } y \in Y .
\end{aligned}
$$

This implies that $d\left(x, y_{0}\right) \leq d(x, Y)+\varepsilon$ i.e. $y_{0} \in L_{Y}(x, \varepsilon)$.

Remarks. If $Y$ is a subspace of a linear metric space $(X, d)$ with a translation invariant metric $d$ and $x_{0}=0$, the additive identity of $X$, then one can choose the function $f$ in the preceeding discussion such that $f \in X^{v}$, where

$$
X^{v}=\left\{f: X \longrightarrow \mathbb{R}: \sup _{x \in X \backslash\{0\}} \frac{|f(x)|}{d(x, 0)}<\infty, f(0)=0, f \text { subadditive }\right\}
$$

is the cone of subadditive functions in $X_{0}$ (see [5] or [6]). If $X$ is a normed linear space then $f \in X^{*}$ (see [7]).

\section{References}

[1] R.C. Buck; Approximation of functions (Ed. H.L. Garabedian) Elsevier, Amsterdam (1965), 27-42.

[2] J.A. Johnson, "Banach spaces of Lipschitz functions and vector-valued Lipschitz functions", Trans. Amer. Math. Soc. 148(1970), 147-169.

[3] Costica Mustăta, "On the best approximation in metric spaces, Mathematika-Revue d'Analyse Numerique et de The'orie de l' Approximation", L'Analyse Numerique et la The'orie de L'Approximation, 4(1975), 45-50.

[4] Costica Mustăta, "A characterization of semi-Chebyshev sets in a metric space, Annal. Numer. Theory Approximation, 7(1978), 169-170.

[5] T.D. Narang and Swaran Khanna, "On some approximation problems in metric linear spaces, Indian J. Pure Appl. Math. 14(1983), 253-256.

[6] G. Pantelidis, "Approximationstheorie für metrische linear Raume, Math. Ann. 184(1969), 30-48.

[7] Ivan Singer, Best approximation in normed linear spaces by elements of linear subspaces, SpringerVerlag, Berlin(1970).

Department of Mathematics, Guru Nanak Dev University, Amritsar - 143005 (India). 